\title{
An Optimal Distribution Model of Emergency Materials Based on Disaster Weather
}

\author{
Guanghui Wang* \\ Chinese Academy of Meteorological Sciences, Beijing 100081, China \\ *Corresponding author: ghwang@cma.gov.cn
}

Received July 22, 2019; Revised August 23, 2019; Accepted September 05, 2019

\begin{abstract}
Effective support of emergency materials is a necessary prerequisite for post-disaster emergency rescue. The transportation and distribution of post-disaster emergency materials includes two stages: from storage warehouses and material distribution centers outside the disaster area to emergency distribution centers outside the disaster area, and from emergency distribution centers to rescue points in the disaster area. Emergency material support has the characteristics of urgent demand and relative shortage of materials. Especially, the transportation of materials from supply points outside the disaster-stricken areas to emergency material distribution centers, along the way, affected by the actual traffic capacity and meteorological conditions, will have a significant impact on the efficient distribution of emergency materials. This paper deals with the optimization of transportation allocation from emergency material supply points to emergency material distribution centers in the periphery of disaster areas. Based on the factors affecting transportation efficiency such as road resistance parameters, attenuation coefficient, and disaster intensity, an optimal allocation model of emergency materials is established, which minimizes the sum of transportation cost, construction cost of distribution center, and penalty cost of transportation time. The validity and feasibility of the model are analyzed and studied by an example. The experimental results show that the attenuation coefficient of the transportation line and the disaster intensity of the road section have important influence on the emergency material allocation scheme. The emergency material allocation scheme formulated by the optimization model is scientific and reasonable.
\end{abstract}

Keywords: emergency supplies distribution, distribution centre, attenuation coefficient, road disaster intensity, optimal location

Cite This Article: Guanghui Wang, "An Optimal Distribution Model of Emergency Materials Based on Disaster Weather." American Journal of Electrical and Electronic Engineering, vol. 7, no. 3 (2019): 83-90. doi: 10.12691/ajeee-7-3-5.

\section{Introduction}

Natural disasters pose a serious threat to the people's life and property security. The key to effectively alleviate the disaster situation and reduce the economic losses in the disaster area is to deliver the emergency materials to the victims quickly and reasonably. How to allocate the rescue materials scientifically, avoid the high risk in the transportation process, and satisfy the material demand of the disaster victims to the maximum extent is an important issue in the post-disaster emergency material distribution. According to the disaster situation, scientific selection of transportation routes and stable distribution center is the premise to ensure the smooth development of assistance work.

The location of post-disaster emergency material distribution center and the distribution of material transportation are the research hotspots of scholars in recent years. The logistics department of emergency materials after disaster is the same as the general social logistics; its process has the characteristics of sudden occurrence, timeliness and fairness. After a disaster occurs, the first consideration for emergency material distribution is to transport emergency materials from emergency storage to emergency material distribution centers located near the disaster area under various constraints such as limited time, space and resources. Then each distribution center will be distributed to each assistance point. Therefore, the location of distribution center and the planning of transportation route are two key problems in emergency material distribution. In addition, after the disaster, the budget funds used to seek assistance are also limited, and the operating cost is also an important factor affecting the distribution of emergency materials. Most research on emergency material distribution is to minimize the cost of transportation of emergency materials as an efficiency goal (e.g. [1,2]). Due to the unsatisfied demand proportion of each rescue point and the delay in distribution time, the social cost will increase. Therefore, there is a multi-objective optimization model for emergency material distribution, which takes the cost of transportation and punishment as the objective function, giving consideration to the efficiency and fairness of the distribution of emergency materials (e.g. [3]). Similar 
works are as follows: a bilevel programming model with the minimum total time consumed by emergency logistics system as the upper goal and the sum of distribution cost and time as the lower level goal was studied in [4]. In reference [5], a multi-objective programming model for the location of emergency material distribution center and the optimization of transportation distribution route was established, which aimed at the timeliness of emergency material support and the fairness of distribution. Reference [6] comprehensively considered the randomness of the travel time of emergency vehicles caused by the location of the request point and the terrain, the uncertainty of the demand for emergency materials and the urgency of the time of distribution of emergency materials, In the paper, a multi-objective fuzzy path optimization model based on opportunity constrained programming was established, aiming at the shortest total time spent on emergency materials and the minimum total distribution cost. In reference [7], a multi-objective, multi-mode, multicommodity, multi-period stochastic model was proposed to guide the allocation of post-disaster emergency materials by means of cost analysis.

When optimizing the location of distribution centers in many literatures, the cost minimization objective function seldom considers the influence of actual road conditions and meteorological factors on transportation efficiency. In the process of transporting emergency materials to alternative distribution centers in disaster areas, the traffic flow information and real-time weather factors of transportation routes have a very important impact on the effective distribution of emergency materials. From meteorological service information provided by meteorological department, decision-makers can choose transportation route and distribution center correctly through different transportation ways and meteorological conditions so as to ensure the rapid and scientific arrival of emergency materials in disaster areas. This paper studies the location problem of emergency material distribution center under the influence of actual transportation conditions and meteorological factors. The minimized cost objective function takes into account the traffic flow of transport lines, the attenuation coefficient affected by disaster weather, and the impact of disaster intensity factor on transportation cost and the time penalty cost of transportation delay due to meteorological factors.

\section{Problem Description and Basic Hypothesis}

\subsection{Problem Description}

After the disaster, the distribution center is usually located around the disaster area to facilitate the distribution of emergency relief materials to specific rescue points. As a result, there is no need for close links between distribution centers. In this paper, we consider setting up several distribution centers on the periphery of the disaster area. The emergency materials storage stores or suppliers in other parts of the country deliver emergency materials to the distribution center first according to the demand, and then from one distribution center to the specific help point. The process is shown in Figure 1.

\subsection{Basic Assumption}

Taking into account the distribution characteristics of post-disaster emergency supplies, the following assumptions are made:

1. Distribution centers select transport hub points that are affected by and within a shorter distance from disaster-affected areas.

2. Regardless of the chain reaction of natural disasters and the occurrence of secondary disasters, the meteorological conditions and disaster conditions in the affected areas are relatively stable.

3. The weather conditions and traffic flow between emergency storage point or supplier to distribution center are stable and the speed is uniform.

4. The emergency materials are shipped from the delivery point to the destination by means of vehicle loading and unloading without stoppage.

5. Each distribution center can accept goods from multiple emergency supply points at the same time and can also deliver goods to multiple demand points at the same time. But distribution centers do not supply each other.

6. The demand for all rescue points can be met, and all goods in the distribution center are used exclusively for rescue point distribution.

\section{Model Construction}

\subsection{Symbol Description}

(I) Set parameters

G: set of emergency material stores or supply points; $\mathrm{M}$ : distribution center alternative address set; C: set of disaster points.

(II) Path parameters

$x_{k i}$ : volume of traffic from the $k$ supply point to the $i$ distribution center; $y_{i j}$ : distribution volume from the $i$ distribution center to the $j$ disaster site; $t_{k i}$ : unit transport costs from the $k$ th supply point of a standard cargo to the $i$ distribution center under the conditions of free movement of transport vehicles; $c_{i j}$ : unit distribution cost of the $i$ distribution center of a standard cargo to the $j$ disaster site; $v_{0}$ : maximum transportation speed from all supply points to each distribution center under free driving conditions. (III) Demand parameter

$D_{j}$ : demand for emergency supplies at the $j$ disaster sites; $f_{i}$ : fixed cost of the $i$ distribution center for one standard cargo; $\rho_{i}$ : variable cost factor for distribution center $I ; A_{k}$ :total supply capacity at the $k$ supply point; $M_{i}$ : maximum capacity of distribution center $I$; $C F_{i}$ : indicates the penalty cost of delivery of emergency supplies from the $k$ supply point to the $i$ distribution center beyond the expected time $E T_{i} ; \delta$ : represents unit material penalty cost. 


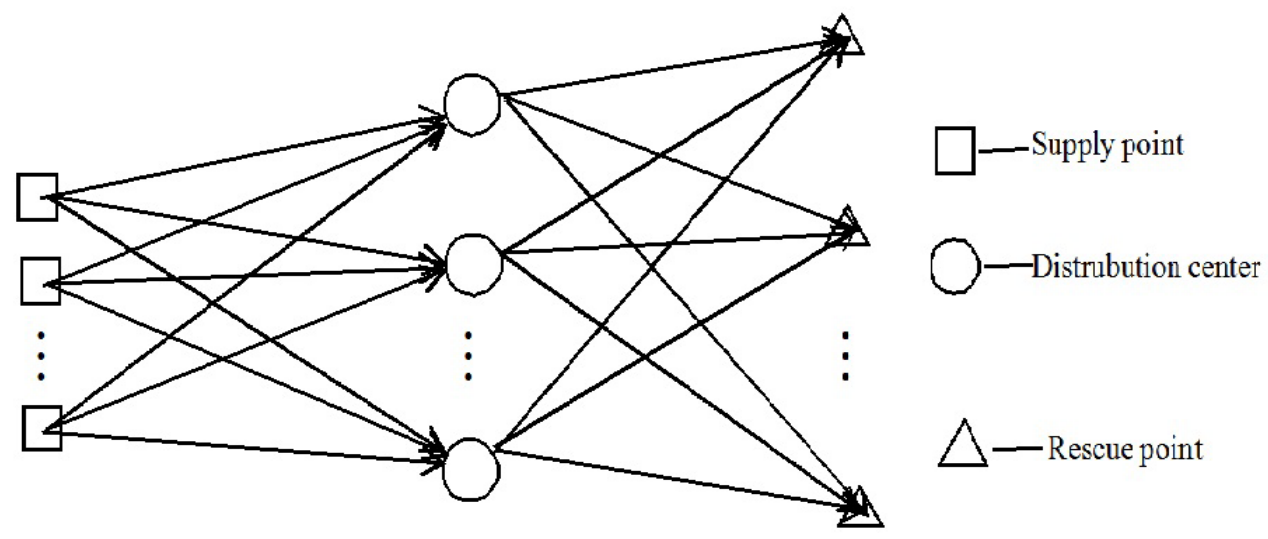

Figure 1. Emergency commodity allocation route schematic drawing

\subsection{Model Construction}

(I) Object function

$$
\begin{aligned}
\min T C & =\sum_{i \in M} \sum_{k \in G} t_{k i} x_{k i}+\sum_{i \in M} \sum_{j \in C} c_{i j} y_{i j} \\
& +\sum_{i \in M} \sum_{k \in G} \rho_{i} x_{k i}+\sum_{i \in M} C F_{i}+\sum_{i \in M} f_{i} z_{i}
\end{aligned}
$$

(II) Constraint condition

$$
\begin{gathered}
\sum_{i \in M} x_{k i} \leq A_{k}, \forall k \in G \\
\sum_{i \in M} y_{i j} \geq D_{j}, \forall j \in C \\
\sum_{k \in G} x_{k i}=\sum_{j \in C} y_{i j}, \forall i \in M \\
\sum_{k \in G} x_{k i} \leq M_{i} z_{i}, \forall i \in M \\
z_{i}=\left\{\begin{array}{l}
\sum_{i \in M} z_{i} \leq n \\
1,
\end{array} \quad \text { If the selected point } i\right. \text { is selected } \\
0, \quad \text { otherwise }
\end{gathered}
$$

among them: the objective function TC in formula (1) is the total cost of emergency material distribution, including: total transportation cost, total distribution cost, fixed cost of building distribution center, total cost of distribution center time punishment, and the variable cost of distribution center (mainly considering the processing cost before emergency materials are sent to the disaster site), are used to express the efficiency objective of the model. Formula (2) indicates that the quantity of emergency supplies provided from supply point $\mathrm{k}$ to distribution center. Formula (3) means that the needs of all rescue points can be met. Type (4) means to ensure that emergency supplies in distribution are exclusively used for rescue point distribution and will not remain. Type (5) indicates the capacity limit of the distribution center. Formula (6) indicates that the number of distribution centers does not exceed alternative addresses. Formula (7) indicates that the transportation costs of emergency materials from supply point to distribution center and from distribution center to disaster point cannot be negative.

Based on the assumption that the meteorological conditions and traffic conditions in the affected area are relatively stable, the unit distribution cost from the distribution center to the disaster site can be a constant. Considering that the distance between the supply point and the distribution center is relatively distant, that the distribution may be more dispersed and that the traffic conditions and meteorological conditions of the transport routes are quite different, Therefore, the transportation cost from supply point to distribution center will increase with the increase of traffic flow and the influence of meteorological factors.

Assuming that the free travel time from the $k$ supply point to the $i$ distribution center is $t t_{k i}^{0}$, the motor vehicle traffic is $Q_{k i}$, and the actual traffic capacity is $P_{k i}$, then the time for the emergency material to reach the distribution center is (e.g.[8])

$$
T_{k i}^{0}=t t_{k i}^{0}\left[1+\alpha\left(Q_{k i} / P_{k i}\right)^{\beta}\right]
$$

where $\alpha, \beta$ is the roadblock parameters.

The transportation efficiency of emergency materials from supply point to distribution center is not only affected by road traffic flow, but also restricted by meteorological conditions. The maximum speed of transportation from the kth supply point to the ith distribution center is $v_{k i}^{0}$ in the absence of disaster weather, and the maximum speed of transportation after disaster weather is as follows (e.g.[9])

$$
v_{k i}=v_{k i}^{0}-\theta \cdot r_{k i}
$$

where $\theta$ is the attenuation factor of the disaster impact, $r_{k i}$ is the disaster intensity of the section from the kth supply point to the ith distribution center. It can be seen from models (9) and (10) that the greater the road traffic volume, the longer the driving time, and the greater the attenuation coefficient and the strength of the road section, the more severe the disaster weather will be, the slower the natural transportation speed will be. The increase of road traffic will increase the transportation cost from the supply point to the distribution center, whether the 
transportation time is prolonged or the transportation speed slows down in the event of disaster weather. Thus, due to the dual effects of vehicle flow and disaster weather, the unit transportation cost of the kth supply point to the ith distribution center is set as

$$
\bar{t}_{k i}=\sigma_{k i} \cdot t_{k i}, \sigma_{k i}=\gamma\left(v_{0}\right)^{\lambda} \cdot\left(v_{k i}\right)^{-\mu}, \gamma \geq 1, \lambda, \mu \geq 0 \text { (11) }
$$

where $\sigma_{k i}$ is a proportional coefficient and $\gamma, \lambda, \mu$ is a parameter, which can be determined by regression method. If we take $T_{k i}^{0}$ as the expected point in time for the emergency supplies in the ith distribution center, then the time penalty cost function is

$$
C F_{i}=\delta \sum_{k \in G} T_{k i}^{0} \frac{\theta \cdot r_{k i}}{v_{k i}^{0}-\theta \cdot r_{k i}} x_{k i} .
$$

The penalty function contains the demand of the distribution center, and there is a linear positive correlation, which is consistent with the practical significance. If the transportation route from the supply point to a distribution center suffers from severe meteorological disasters, the corresponding penalty cost will increase abruptly. The $C F_{i}$ in formula (1) is replaced by (12), then the mathematical model is a mixed integer linear programming problem with constraint, which takes the total cost of emergency material distribution as the minimum objective.

$$
\begin{gathered}
\min T C=w_{1}^{T} \xi+w_{2}^{T} z \\
\text { S.t. } A_{1} \xi+B_{1} z \leq b_{1} \\
A_{2} \xi+B_{2} z=b_{2} \\
\xi \geq 0, \xi \in X \subset R^{S+n}, z \in\{0,1\}
\end{gathered}
$$

where

$$
\xi=\left(\begin{array}{l}
x_{11}, x_{12}, \cdots, x_{1 n}, \cdots, x_{s 1}, x_{s 2}, \cdots, x_{s n}, \\
y_{11}, y_{12}, \cdots, y_{1 d}, \cdots, y_{n 1}, y_{n 2}, \cdots, y_{n d}
\end{array}\right)^{T},
$$

$z=\left(z_{1}, z_{2}, \cdots z_{n}\right)$. The subscript corresponds to the serial number of the supply point, distribution center, and rescue point in the known data sheet. Furthermore, the optimization problem (14) (17) can be transformed into the following standard form:

$$
\begin{gathered}
\min T C=\bar{L} \cdot \vec{X} \\
\text { s.t. } B \cdot \vec{X} \leq \vec{b}, \text { Aeq } \cdot \vec{X}=\text { beq, } \vec{X} \geq 0
\end{gathered}
$$

Where $\vec{X}=(\xi, z)^{T}, \quad \vec{L}, B, \vec{b}$, Aeq, beq calculated from known parameters. The coefficient matrix and vector in the optimization problem (18) (19) are substituted into the Matlab7.0 integral linear programming library function:

$$
\begin{aligned}
& {[X, \text { fval, flag }]} \\
& =\text { int linprog }(\vec{h}, s \times n+n \times d+n, G, \vec{b}, \text { Aeq, beq, } 0,[]) ;
\end{aligned}
$$

the minimum value fval of the objective function TC and the optimal solution $\vec{X}$ of the optimization model (18) (19) are obtained by running, and then the optimal solution $\left(x_{k i}\right),\left(y_{i j}\right)$ and $\left(z_{i}\right)$ of the optimization model $(1) \sim(8)$ are obtained from $\vec{X}$.

\section{Numerical Example and Analysis}

\subsection{Example and Parameter Setting}

It is assumed that there are 4 alternative emergency distribution centers in the periphery of the disaster area, 8 Rescue Points $(R P)$ inside and 4 emergency supply points outside the disaster area. Under the condition of material quantity, time limit and meteorological influence, the location of distribution center and emergency supply point are optimized. Without loss of generality, set the maximum transportation speed $v_{0}=90 \mathrm{~km} / \mathrm{h}$ under free driving conditions, unit material penalty cost $\delta=1$. The delivery time from the $k$ th emergency Supply Point (SP) to the ith Distribution Centre $(D C)$ is $t t_{k i}^{0}$ (see Table 1) and the unit transportation cost of a standard cargo $t_{k i}$ (see Table 2). The remaining known parameters are shown in Table 3-Table 5.

Table 1. Transportation Time under Free Driving Conditions (Unit: hour)

\begin{tabular}{|c|c|c|c|c|}
\hline $\begin{array}{c}\text { SP } \\
\text { DC }\end{array}$ & DC1 & DC2 & DC3 & DC4 \\
\hline SP1 & 6.23 & 6.8 & 7.6 & 7.75 \\
\hline SP2 & 6.57 & 6.45 & 7 & 5.23 \\
\hline SP3 & 7.5 & 7.4 & 7.86 & 6 \\
\hline SP4 & 5.2 & 4.48 & 4.67 & 4.38 \\
\hline
\end{tabular}

Table 2. Transportation Costs under Free Driving Conditions

\begin{tabular}{|c|c|c|c|c|}
\hline $\begin{array}{c}\text { SP } \\
\text { DC }\end{array}$ & DC1 & DC2 & DC3 & DC4 \\
\hline SP1 & 6.096 & 7.112 & 8.128 & 8.128 \\
\hline SP2 & 6.096 & 7.112 & 6.096 & 4.064 \\
\hline SP3 & 8.128 & 8.128 & 7.112 & 6.096 \\
\hline SP4 & 8.128 & 7.112 & 5.080 & 6.096 \\
\hline
\end{tabular}

Table 3. Distribution Cost and Rescue Point Demand

\begin{tabular}{|c|c|c|c|c|c|c|c|c|}
\hline $\begin{array}{c}\text { DC } \\
\text { RP }\end{array}$ & RP1 & RP2 & RP3 & RP4 & RP5 & RP6 & RP7 & RP8 \\
\hline DC1 & 1.5 & 1.2 & 1.2 & 1.8 & 1.5 & 2.4 & 2.1 & 1.8 \\
\hline DC2 & 2.4 & 1.8 & 2 & 2.7 & 2.1 & 1.8 & 1.5 & 2.4 \\
\hline DC3 & 2.4 & 2.1 & 2.1 & 3.2 & 2.4 & 1.2 & 1 & 0.8 \\
\hline DC4 & 2.7 & 2.4 & 2.1 & 3.2 & 3 & 3.2 & 2.7 & 2.4 \\
\hline$D_{j}$ & 360 & 420 & 450 & 220 & 160 & 580 & 480 & 520 \\
\hline
\end{tabular}

Table 4. Fixed investment costs, variable costs and maximum capacity of distribution centers

\begin{tabular}{|c|c|c|c|c|}
\hline & DC1 & DC 2 & DC3 & DC4 \\
\hline Fixed investment cost $f_{i}$ & 1860 & 1520 & 1640 & 1340 \\
\hline variable expenses $\rho_{i}$ & 0.65 & 0.46 & 0.52 & 0.37 \\
\hline maximum capacity $M_{i}$ & 1620 & 2430 & 2080 & 1850 \\
\hline
\end{tabular}


Table 5. Capacity of supply points to supply emergency supplies

\begin{tabular}{|c|c|c|c|c|}
\hline & SP1 & SP2 & SP3 & SP4 \\
\hline Supply capacity $A_{k}$ & 600 & 700 & 1100 & 1000 \\
\hline
\end{tabular}

\subsection{Parameter Sensitivity Test}

The background of the experiment is that the normal section from the supply point to the distribution center encounters disaster weather. Because the actual capacity and the traffic volume of the transportation routes from each supply point to the alternative distribution center are different, the ratio $Q_{k i} / P_{k i}=\Gamma_{k i}$ will vary with the different transportation lines. Take $\alpha=0.15, \beta=4$. $r_{k i}$, $\Gamma_{k i}$ and randomly assigned in the range of $(0,1)$; the undetermined parameter in formula (11) is $\gamma=\lambda=\mu=1$.

\subsubsection{Sensitivity Test of Ratio $\Gamma_{k i}$}

We test the effect of the change of the ratio $\Gamma_{k i}$ of the traffic condition on the optimization scheme of emergency material distribution. Take attenuation coefficient $\theta=80$. Random values of disaster intensity $\left(r_{k i}\right)$ and ratio $\left(\Gamma_{k i}\right)$ of road sections are as follows:

$$
\begin{aligned}
\left(r_{k i}\right) & =\left(\begin{array}{llll}
0.8055 & 0.8865 & 0.9787 & 0.0596 \\
0.5767 & 0.0287 & 0.7127 & 0.6820 \\
0.1829 & 0.4899 & 0.5005 & 0.0424 \\
0.2399 & 0.1679 & 0.4711 & 0.0714
\end{array}\right), \\
\left(\Gamma_{k i}\right) & =\left(\begin{array}{llll}
0.3517 & 0.9172 & 0.3804 & 0.5308 \\
0.8308 & 0.2858 & 0.5678 & 0.7792 \\
0.5853 & 0.7572 & 0.0759 & 0.9340 \\
0.5497 & 0.7537 & 0.0540 & 0.1299
\end{array}\right),
\end{aligned}
$$

get calculation results: $z=(0,1,0,1)$,

$$
\begin{aligned}
\left(x_{k i}\right) & =\left(\begin{array}{rrrr}
0 & 0 & 0 & 390 \\
0 & 700 & 0 & 0 \\
0 & 0 & 0 & 1100 \\
0 & 640 & 0 & 360
\end{array}\right), \\
\left(y_{i j}\right) & =\left(\begin{array}{rrrrrrrr}
0 & 0 & 0 & 0 & 0 & 0 & 0 & 0 \\
0 & 120 & 0 & 0 & 160 & 580 & 480 & 0 \\
0 & 0 & 0 & 0 & 0 & 0 & 0 & 0 \\
360 & 300 & 450 & 220 & 0 & 0 & 0 & 520
\end{array}\right) .
\end{aligned}
$$

The row number of the matrix $\left(r_{k i}\right),\left(\Gamma_{k i}\right),\left(x_{k i}\right)$ is the serial number of the supply point and the column number is the serial number of the distribution center; the component serial number of the $z$ is the distribution center serial number. The serial number of the row in the matrix $\left(y_{i j}\right)$ corresponds to the distribution center serial number, and the serial number of the column corresponds to the rescue point serial number.

Therefore, the above calculation results are as follows: (a) the location of distribution center is obtained from the component value of $\mathrm{z}$ : distribution center 2, distribution center 4; (b) from matrix $\left(x_{k i}\right)$, it is known that distribution center 4 receives 390 supplies from supply point 1 ; distribution center 2 receives 700 supplies from supply point 2, and distribution center 4 receives 1100 supplies from supply point 3 , distribution center 2 and distribution center 4 obtain 640 and 360 supplies from supply point 4, respectively; (c) from matrix A: distribution center 2 provides 120, 160, 580, 480 relief materials to rescue points $2,5,6$ and 7 respectively; distribution center 4 provides 360, 300, 450, 220, 520 relief materials to rescue points $1,2,3,4$ and 8 respectively.

When the values of attenuation coefficient $\theta$ and road disaster intensity $\left(r_{k i}\right)$ are determined, it is proved by many experiments that the random value of ratio $\Gamma_{k i}$ cannot change the result of distribution center location, and the change of supply and distribution quantity is also very small. It shows that ratio $\Gamma_{k i}$ has little effect on distribution center location and distribution plan. As to why such a result will occur, further study is needed on the parameters of formula (9) and the structure of vehicle traffic on the road section.

The influence of attenuation coefficient directly related to disaster weather and disaster intensity of road section on distribution center location and emergency material distribution scheme is discussed below.

\subsubsection{Attenuation Coefficient $\theta$}

When the ratio $\Gamma_{k i}$ and the disaster intensity $r_{k i}$ of the road section are determined, the location and distribution plan of the distribution center will also change with the change of $\theta$. The random values of ratio $\Gamma_{k i}$ and road disaster intensity $r_{k i}$ are shown in formula (20), then

When $\theta=40, z=(0,1,1,1)$

$$
\begin{aligned}
\left(x_{k i}\right) & =\left(\begin{array}{rrrr}
0 & 0 & 0 & 390 \\
0 & 700 & 0 & 0 \\
0 & 0 & 0 & 1100 \\
0 & 0 & 1000 & 0
\end{array}\right), \\
\left(C F_{k i}\right) & =\left(\begin{array}{lrrr}
3.4945 & 5.8107 & 5.9019 & 0.2160 \\
2.6652 & 0.0835 & 3.3716 & 2.5957 \\
0.6883 & 2.2994 & 2.2486 & 0.1434 \\
0.6388 & 0.3986 & 1.2367 & 0.1436
\end{array}\right),
\end{aligned}
$$

When $\theta=80, z=(0,1,0,1)$.

$$
\begin{aligned}
\left(x_{k i}\right) & =\left(\begin{array}{rrrr}
0 & 0 & 0 & 390 \\
0 & 700 & 0 & 0 \\
0 & 0 & 0 & 1100 \\
0 & 640 & 0 & 360
\end{array}\right), \\
\left(C F_{k i}\right) & =\left(\begin{array}{rrrr}
15.8706 & 51.0831 & 52.2595 & 0.4442 \\
8.5780 & 0.1692 & 12.8261 & 9.8010 \\
1.5129 & 6.5335 & 6.2994 & 0.2930 \\
1.4537 & 0.8712 & 3.3645 & 0.2968
\end{array}\right)
\end{aligned}
$$

When $\theta=40$, from the vector $z$ we know distribution center 2, 3, 4 are selected. The matrix $\left(x_{k i}\right)$ in formula 
(22), the $x_{14}=390, x_{22}=700, x_{34}=1100, x_{43}=1000$ are the supply point $1,2,3,4$ to the selected distribution center $4,2,4,3$ to provide the quantity of emergency supplies respectively. According to the road section disaster intensity matrix $\left(r_{k i}\right)$ in formula (20), the corresponding $r_{14}=0.0596, r_{22}=0.0287, r_{34}=0.0424$, $r_{43}=0.4711$ are the least in the column, indicating that the disaster intensity between the supply point and the selected distribution center is the least, which accords with the basic requirement of optimizing the location of distribution center. In addition, in matrix $\left(C_{k i}\right)$ in formula (20), the time penalty costs indicated by $C F_{14}=0$. 2160, $C F_{22}=0.835, C F_{34}=0.1434, C F_{43}=0.1436$, except $C F_{43}=0.1436$, are all the least in the column. Because the time penalty cost is only a part of the total cost, it is normal to show that the individual time cost penalty cost is not optimal.

Where $\theta=80$, similar to the analysis above, the selected distribution center is: distribution center 2, 4. Supply points $1,2,3,4$, and 4 provide 390, 700, 1100, 640, 360 emergency supplies to distribution centers 4, 2, 4, 2 and 4, respectively. In matrix $\left(r_{k i}\right)$ of formula (20), $r_{14}, r_{34}, r_{44}$ and $r_{22}, r_{42}$ are the smallest in the column, and in matrix $\left(C F_{k i}\right)$ of formula (23), $C F_{14}, C F_{34}, C F_{44}$ and $C F_{22}, C F_{42}$ are the smallest in the column, which meets the requirement of optimal location of distribution center.

Based on formulas (10) and $v_{k i} \geq 0$, then there are $0 \leq \theta \leq v_{k i}^{0} / r_{k i}$. In the range of $\theta$, given $\left(r_{k i}\right)$ in formula (20), the value of $\theta$ has the following effects on the location of distribution centers (see Table 6).

Table 6. Effect of Attenuation Coefficient Change on Distribution Center Location

\begin{tabular}{|c|c|c|c|c|c|}
\hline$\theta$ & 0 & 10 & 20 & 30 & 40 \\
\hline DC & $1,3,4$ & $1,3,4$ & $1,3,4$ & $1,2,3,4$ & $2,3,4$ \\
\hline$\theta$ & 50 & 60 & 70 & 80 & 90 \\
\hline DC & $2,3,4$ & $2,3,4$ & $2,3,4$ & 2,4 & 2,4 \\
\hline
\end{tabular}

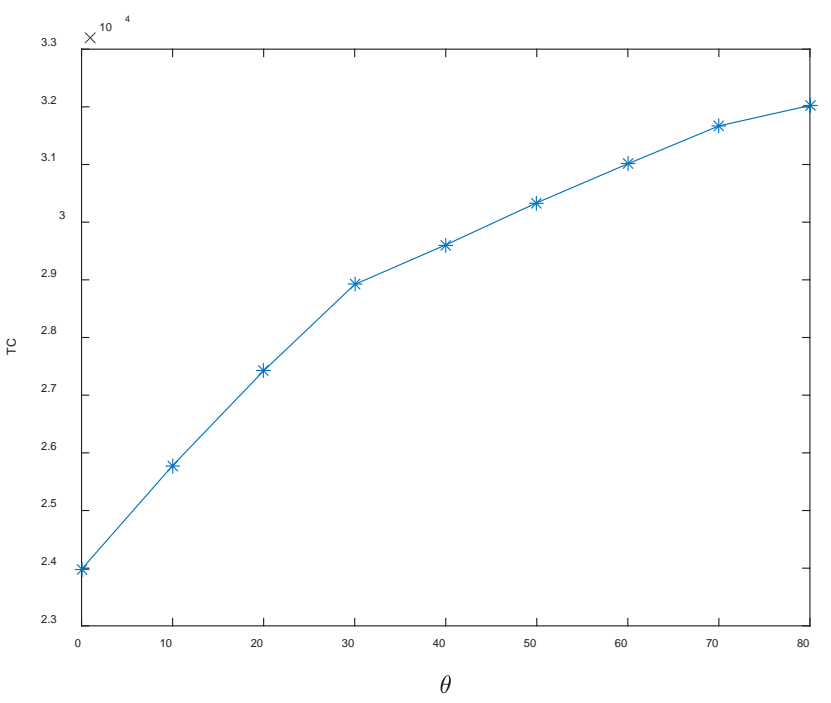

Figure 2. Attenuation coefficient $\theta$ versus total distribution cost TC

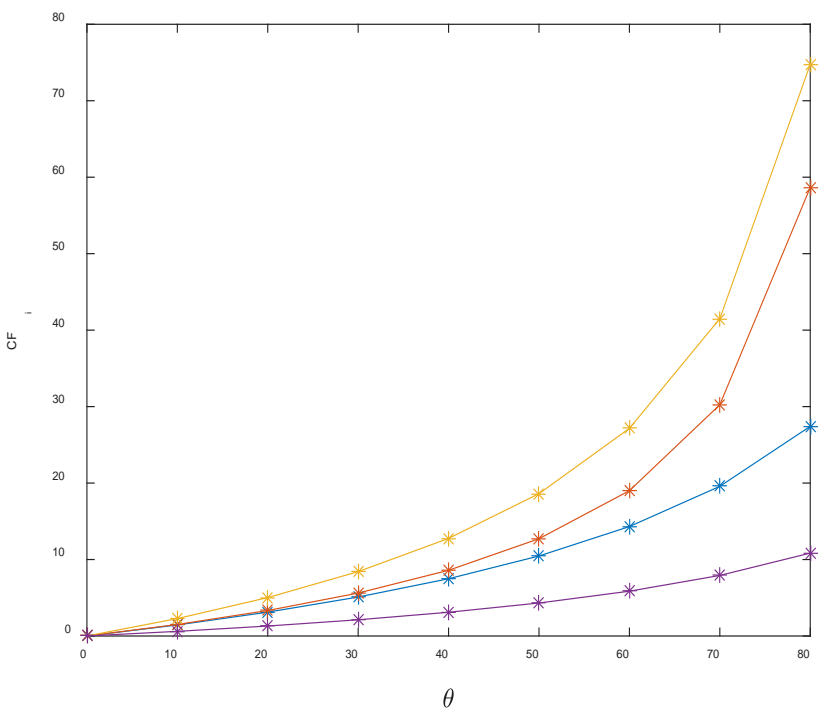

Figure 3. Attenuation Coefficient $\theta$ and Time Penalty Cost Diagram

Figure 2 shows an almost linear incremental relationship between the attenuation coefficient and the total cost. The increase of attenuation coefficient leads to the increase of time penalty cost, which makes the total transportation cost of emergency materials increase rapidly.

Figure 3 shows the increasing relationship between the attenuation coefficient and the time penalty cost of four alternative distribution centers. The curves in the graph correspond to distribution center 3 , distribution center 2, distribution center 1 and distribution center 4 in turn from top to bottom, which shows that the order of time penalty cost of four alternative distribution centers will not change with the change of $\theta$.

\subsubsection{Strength of Road Section Disaster $r_{k i}$}

When the attenuation coefficient $\theta=60$ and the ratio matrix $\left(\Gamma_{k i}\right)$ is shown in equation (20), the location scheme of distribution center will change with the change of disaster intensity $\left(r_{k i}\right)$. The following two groups of road disaster intensity values $\left(r_{k i}\right)_{1}$ and $\left(r_{k i}\right)_{2}$ are tested.

$$
\begin{aligned}
\left(r_{k i}\right)_{1} & =\left(\begin{array}{llll}
0.5439 & 0.2187 & 0.4046 & 0.6279 \\
0.7210 & 0.1058 & 0.4484 & 0.7720 \\
0.5225 & 0.1097 & 0.3658 & 0.9329 \\
0.9937 & 0.0636 & 0.7635 & 0.9727
\end{array}\right), \\
\left(r_{k i}\right)_{2} & =\left(\begin{array}{llll}
0.0445 & 0.6878 & 0.6834 & 0.3309 \\
0.7549 & 0.3592 & 0.7040 & 0.4243 \\
0.2428 & 0.7363 & 0.4423 & 0.2703 \\
0.4424 & 0.3947 & 0.0196 & 0.1971
\end{array}\right) .
\end{aligned}
$$

When the strength of section $\left(r_{k i}\right)_{1}$ in (24) is chosen, the experimental results are as follows: $z=(0,1,1,0)$

$$
\left(x_{k i}\right)=\left(\begin{array}{rrrr}
0 & 390 & 0 & 0 \\
0 & 700 & 0 & 0 \\
0 & 340 & 760 & 0 \\
0 & 1000 & 0 & 0
\end{array}\right)
$$




$$
\left(C F_{k i}\right)=\left(\begin{array}{cccc}
3.5649 & 1.4462 & 2.8279 & 5.7629 \\
7.4764 & 0.4905 & 3.0989 & 6.5608 \\
4.1907 & 0.6454 & 2.5352 & 15.0824 \\
10.7772 & 0.2185 & 4.8413 & 8.0820
\end{array}\right) .
$$

Distribution center location is distribution centers 2 and 3. From the matrix ( $x_{k i}$ ) of (25), supply points 1, 2, 3, 4, respectively, supply 390, 700, 340, 1000 to distribution center 2, and supply points 3 also supply 760 to distribution center 3 . In comparison with the link disaster intensity matrix $\left(r_{k i}\right)_{1}$ in (24) and the time penalty cost matrix $\left(C F_{k i}\right)$ in (25), the second column of the matrix is smaller than the value on the other columns, and the value of the element in the third row and the third column is the smallest in the third column. The distribution center with low disaster intensity and low cost of time penalty meets the requirements of optimal location. Alternative distribution centers 1 and 4 were eliminated because the sum of columns 1 and 4 in A was much larger than the sum of the other two elements, indicating that the sum of time penalty costs for alternative distribution centers 1 and 4 is too large to be eliminated.

When the strength of section $\left(r_{k i}\right)_{2}$ in (24) is chosen, the experimental results are as follows: $z=(1,0,1,1)$

$$
\begin{aligned}
& \left(x_{k i}\right)=\left(\begin{array}{rrrr}
600 & 0 & 0 & 0 \\
0 & 0 & 0 & 700 \\
0 & 0 & 0 & 890 \\
0 & 0 & 1000 & 0
\end{array}\right), \\
& \left(C F_{k i}\right)=\left(\begin{array}{lrrr}
0.1912 & 7.7419 & 6.4175 & 2.2533 \\
8.2390 & 2.0358 & 6.4756 & 2.3487 \\
1.5049 & 8.2485 & 3.2869 & 1.6790 \\
2.2481 & 1.7894 & 0.0618 & 0.6625
\end{array}\right) .
\end{aligned}
$$

The location of distribution center is distribution center 1,3 , and 4 . The number of emergency supplies provided by supply points $1,2,3,4$ and 4 to distribution center 1, 4, 4 and 3 is 600, 700, 890, 1000, respectively. Compared with B in (24) and (26), the principle of low disaster intensity and low time cost is basically followed by the supply point supplying goods to the distribution center.

The main factors affecting the location of distribution centers are the disaster intensity and attenuation coefficient under disaster meteorological conditions. The smaller the disaster intensity of the section, the lower the time penalty cost from the supply point to the alternative distribution centers, the easier the alternative distribution centers will be selected. Similarly, the time penalty cost and the total cost of distribution will increase with the increase of attenuation coefficient (Figure 2, Figure 3). For the alternative distribution centers with high cost, they will be eliminated naturally. Another phenomenon in the experiment is that when the time penalty cost from the supply point to all members of the alternative distribution is very high, the supply point will also be disqualified when the distribution location is optimized.

\section{Conclusion}

Transportation and distribution of emergency materials is an important part of post-disaster rescue and support work. The optimal selection of distribution centers is very important to reduce property losses and ensure life safety. This paper mainly studies the location optimization of emergency material distribution center under the influence of meteorological factors, focusing on the section transportation from supply point to distribution center. The attenuation coefficient and the disaster intensity of the road section affected by the disaster weather have a very important influence on the selection of distribution centers. The test results show that the change of attenuation coefficient and disaster intensity may cause the change of optimal location scheme of distribution center. Moreover, in the process of optimizing the selection of distribution centers, the demand for supply points is also put forward, and the supply points with higher time penalty cost will be eliminated. It achieves the joint decision-making of optimizing the selection of distribution centers and supply points at the same time, taking the total cost of transportation and distribution as the minimum objective, and provides a scientific basis for formulating the emergency material distribution plan for sudden natural disasters.

\section{Fund}

This research was supported by the National Natural Science Foundation (NSF) of China (No. 91437112).

\section{References}

[1] Haghani A, Oh S C. Formulation and solution of a multicommodity, multimodel network flow model for disaster relief operations, Transportation Research A, 30(3), 231-250, 1996.

[2] Oh S C, Haghani A. Testing and evaluation of a multi-commodity multimodel network flow model for disaster relief management, Journal of Advanced Transportation, 31(3), 249-282, 1997.

[3] Feng Chun, Xiang Yang, et al, Multi-objective Optimization Model of the Emergency Logistics Distribution with Multicycle and Multi-item, Chinese Journal of Management Science, 25(4), 124-132, Apr. 2017.

[4] Lou Zhen-kai, Bi-level Programming Model and Algorithm of Location-routing Problem in Emergency Logistics, Chinese Journal of Management Science, 25(11), 151-157, Nov. 2017.

[5] Qu Chong-chong, Wang Jin, et al, Dynamic Emergency Materials Distribution with Timeliness and Fairness Objective for Post-Earthquake Emergency Rescue, Chinese Journal of Management Science, 26(6), 178-187, Jun. 2018.

[6] Liu Chang-shi, Peng Yi, Kou Gang, Research on Fuzzy Locationrouting Problem in Post-earthquake Delivery of Relief Materials, Chinese Journal of Management Science, 24(5), 111-118, May 2018.

[7] Sheu J B. An emergency logistics distribution approach for quick response to urgent relief demand in disasters, Transportation Research Part E Logistics \& Transportation review, 43(6), 687709, 2007. 
[8] Lam W H K, Shao H, Sumalee A. Modeling impacts of adverse weather conditions on a road network with uncertainties in demand and supply, Transportation Research Part B, 42(10), 890-910, 2008.
[9] Zhu Li, Sang Dan-qiu, Emergency Transportation Path Selection Based on Meteorological Information, China Safety Science Journal, 22(7), 171-176, Jul. 2012. 